\title{
Stage IIIB Eyelid Carcinoma AJCC v8
}

National Cancer Institute

\section{Source}

National Cancer Institute. Stage IIIB Eyelid Carcinoma A/CC v8. NCI Thesaurus. Code C140524.

Stage IIIB includes: Any T, N2, M0. N2: Metastasis in a single ipsilateral regional lymph node, measuring more than $3 \mathrm{~cm}$ in greatest dimension, or in bilateral or contralateral lymph nodes. M0: No distant metastasis. (AJCC 8th ed.) 